\title{
Monitoring of groundwater redistribution in a karst aquifer using a superconducting gravimeter
}

Maxime Mouyen ${ }^{1}$, Laurent Longuevergne ${ }^{2}$, Konstantinos Chalikakis ${ }^{3}$, Naomi Mazzilli $^{3}$, Chloé Ollivier ${ }^{3}$, Séverine Rosat ${ }^{4}$, Jacques Hinderer ${ }^{4}$, and Cédric Champollion ${ }^{5}$.

${ }^{1}$ Department of Space, Earth and Environment, Chalmers University of Technology, Onsala Space Observatory, Onsala, Sweden.

${ }^{2}$ Univ Rennes, CNRS, Géosciences Rennes, UMR 6118, 35000 Rennes, France

${ }^{3}$ Avignon Université, UMR 1114 EMMAH (AU/INRA), Avignon, France

${ }^{4}$ Institut de Physique du Globe de Strasbourg, UMR 7516, Université de Strasbourg/EOST, CNRS, 5 rue Descartes, 67084 Strasbourg, France

${ }^{5}$ Université Montpellier 2 CC 60, UMR 5243 (CNRS/UM2) Géosciences Montpellier, Montpellier, France

\begin{abstract}
Geodetic tools monitor the earth's deformation and gravity field. They are presently sensitive enough to record subtle changes triggered by hydrological processes, thus providing complementary data to standard hydrological measurements. Among these tools, superconducting gravimeter (SG) have proven useful to unravel groundwater redistribution, which significantly alter the gravity field. In the frame of the EquipEx MIGA (Matter wave-laser based Interferometer Gravitation Antenna) project, one SG (iOSG-24) was set up in July 2015 in the Low-noise Underground Laboratory (LSBB) at Rustrel, France, in a gallery located $500 \mathrm{~m}$ beneath the surface. In this work, we analyse the underground iOSG-24 gravity time series together with hydro-meteorological data and basic gravity modelling. We find that the gravimeter recorded the redistribution of water in the ground and that most of this redistribution occurs in the unsaturated zone located above the gravimeter. Nevertheless, residuals between our model and the gravity data suggest the occurrence of large lateral fluxes and rapid runoff not considered in our model. We discuss how the setting of a second SG, planned in July 2018, at the surface of the LSBB could help unravelling such hydrological processes.
\end{abstract}

\section{Introduction}

Superconducting gravimeters (SG) are terrestrial continuous relative gravimeters aiming at recording gravity times-series at the decadal time-scale [1]. Gravity changes being an expression of mass redistribution, SG offer a wide range of application in geodynamics, including tidal analysis [2], earth interior studies [3], fast seismic source determination [4] and assessment of groundwater redistribution [5]; the reader is referred to [6] for a more detailed review. In this work, we analyse the gravity time-series recorded by the SG (iOSG24) set in July 2015 inside the Low-noise Underground Laboratory (LSBB) at Rustrel, France, about $500 \mathrm{~m}$ beneath the surface, in terms of groundwater effect. The LSBB galleries cross arbitrarily the unsaturated zone of the Fontaine de Vaucluse karst hydrosystem [7]. Therefore, it is expected that groundwater redistributions near the SG will be recorded in the gravity time-series, hence providing information on the hydrological processes occurring in this area. One interest is that gravity changes are integrative 
measurements that are not altered by the heterogeneous structure of karst systems (compared to local direct measurements such as those done by, e.g., piezometers). Thus, any water mass redistribution $(\Delta m)$ theoretically changes the gravity $(\Delta g)$. However, according to Newton's universal law of gravitation, if such a mass redistribution occurs at a distance $r$ from the gravimeter, then we have $\Delta \mathrm{g}=\Delta m G / r^{2}$ (G being the universal constant of gravitation). Therefore, using gravity alone does not allow to unequivocally retrieve both the mass of redistributed water and its location, requiring additional constraints from other measurements or assumptions based on the local hydrogeological context [8], [9]. After processing the raw gravity time series from the iOSG-24 we interpret the gravity residuals in terms of groundwater redistribution. Eventually, we discuss the perspectives opened by the setting of a second SG (iGrav031), on the surface, directly above iOSG-24.

\section{Processing of i-OSG24 data}

Gravimetry is an integrative technique that records any gravity change. A targeted use, toward local groundwater monitoring in our case, implies to remove all gravity-contributors not related to local groundwater. Thus, we remove:

1. Tides (body tides and ocean tide loading, [10]). These are by far the largest effect, ranging from -1900 to $1000 \mathrm{~nm} \cdot \mathrm{s}^{-2}$.

2. Polar motion as computed using TSOFT [11] and IERS parameters (ftp://hpiers.obspm.fr/iers/eop/eopc04/), ranging from -10 to $40 \mathrm{~nm} . \mathrm{s}^{-2}$ changes.

3. Atmospheric effects using air pressure data measured at LSBB and a barometric admittance of $-3 \mathrm{~nm} \cdot \mathrm{s}^{-2} \cdot \mathrm{hPa}^{-1}$. This correction accounts for -80 to $50 \mathrm{~nm} \cdot \mathrm{s}^{-2}$ changes.

4. Non-local hydrological effects (that is the gravity changes due to non-local hydrological loading) available from the EOST loading service (http://loading.ustrasbg.fr/ [12]) and computed using the MERRA-2 hydrological model [13]. These effects range within $\pm 15 \mathrm{~nm} \cdot \mathrm{s}^{-2}$.

The instrumental drift of the SG was shown to be negligible three months after the installation [10], so we do not correct it and focus our analysis to data starting from October 2015. Given the location of iOSG-24, no further corrections of slow tectonic processes or global isostatic adjustment are required. The results of these processing steps are given in Figure 1 . 


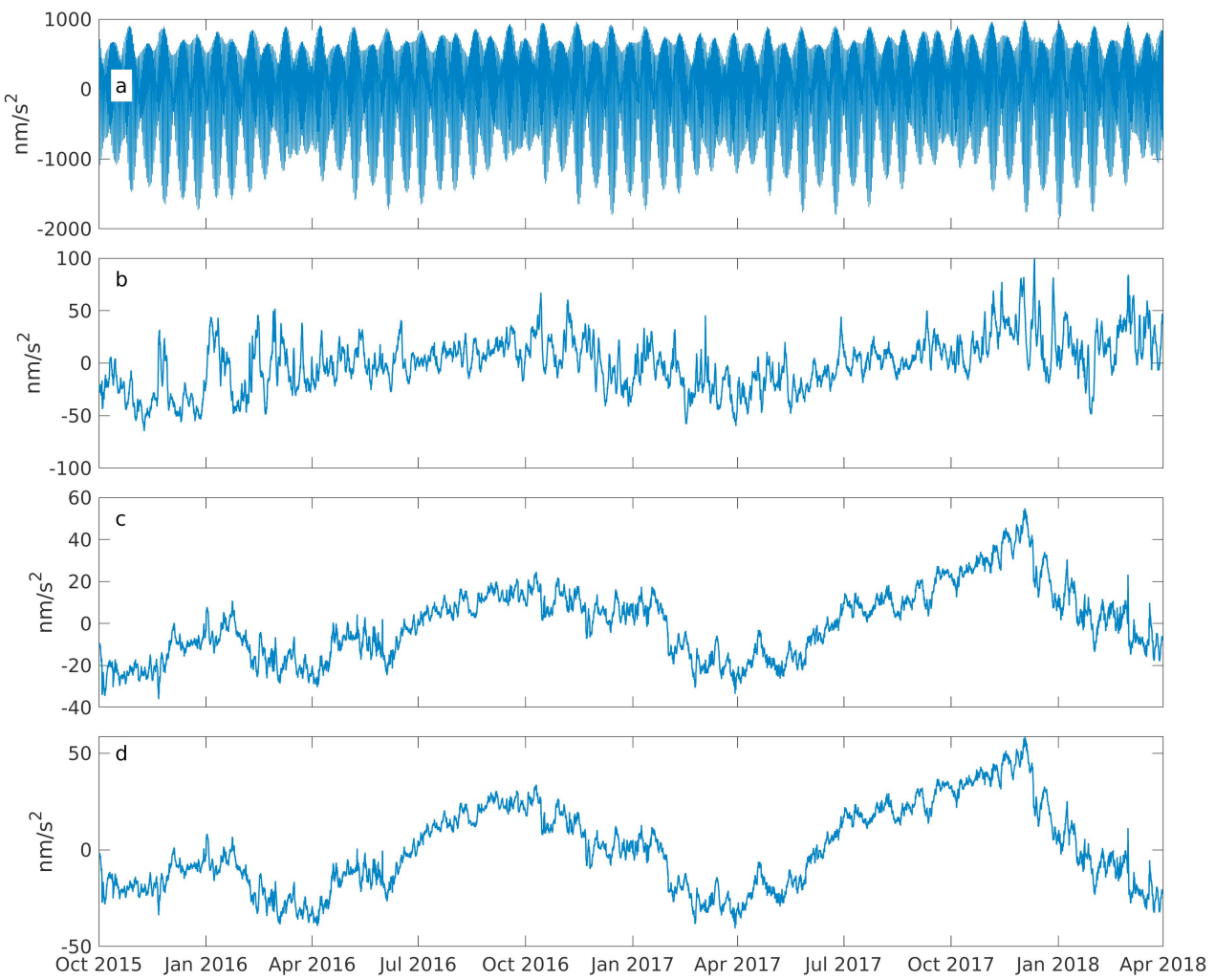

Fig. 1. Processing workflow of the SG data. a) Original iOSG-24 data exhibiting tidal effects on gravity. b) Tides and polar motion effects were removed. c) Air pressure effect was removed. d) Nonlocal hydrological effects were removed.

Figure 1d shows the final gravity residuals, which will be interpreted as groundwater redistribution effects.

\section{Hydro-gravity modelling}

In this part we explain how to combine gravity and hydrological modelling to interpret the iOSG-24 gravity residuals.

\subsection{Gravity modelling}

Although karst systems are complex media, we consider here that most of the water is homogeneously stored as a layer in the matrix porosity of the calcareous rocks. We simply set the constraint that this layer of groundwater should at the first order match the topography. Therefore, we use a digital elevation model centered on the iOSG-24 location as the main geometry of a water layer, which thickness is set here to $10 \mathrm{~cm}$. We then offset this layer at regular intervals from the surface $(510 \mathrm{~m}$ above the gravimeter $)$ toward the depth (down to the saturated zone, $\sim 280 \mathrm{~m}$ below the gravimeter [14]) and compute, in each case, its gravitational effect at the location of the iOSG-24 using right rectangular prisms method [15]. Horizontally, this layer has a circular shape, which radius is increased from 20 to $10000 \mathrm{~m}$. This allows us to evaluate the radius after which the gravity effect reaches an 
asymptote, which means that further water masses are too far to significantly change the gravity effect. This modelling is summarized in Figure 2.
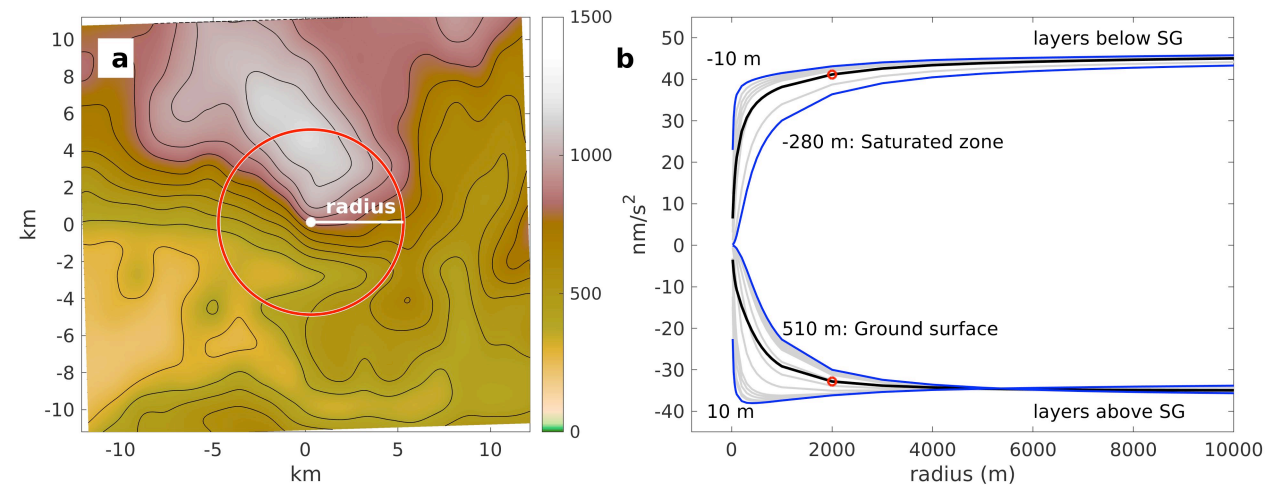

Fig. 2. a) Digital elevation model (in $\mathrm{m}$ ) centered on iOSG-24 (white dot). The red circle illustrates the radius of integration of a hypothetical water layer for computing the gravity effect of this layer at iOSG-24. b) Gravity effects (gray line) of the water layer as a function of its radius and of its location, from $510 \mathrm{~m}$ above the gravimeter (surface) up to $280 \mathrm{~m}$ below, where the saturated zone is supposed to begin, and passing near the gravimeter $(+/-10 \mathrm{~m}$ above or below). The average gravity effect for layers above/below the gravimeter is in black. The red dot shows the radius for which $90 \%$ of the average asymptotic value is reached, numerically:10-cm-water layer above $=-35 \mathrm{~nm} \cdot \mathrm{s}^{-2}$ (that is $3.5 \mathrm{~nm} \cdot \mathrm{s}^{-2} \cdot \mathrm{cm}^{-1}$ ); $10-\mathrm{cm}$-water layer below $=41 \mathrm{~nm} \cdot \mathrm{s}^{-2}$ (that is $4.1 \mathrm{~nm} \cdot \mathrm{s}^{-2} \cdot \mathrm{cm}^{-1}$ ). Note that the ratios in bracket do not change if they are computed with a 1-cm -water layer thickness.

\subsection{Hydrological model}

This model aims at estimating the amount of water stored in the ground as a function of time. Below we briefly describe the principle of this modelling, but all details can be found in [16] and Figure 1 herein. We consider a theoretical model built with two water stores representing a production store and a routing store and determined by four parameters. Part of the water initially entering the ground during rains will flow straight into the production store while the rest of it continues through the ground. The production store then delivers his water, which will join the water already flowing through the ground. This water splits again either toward a deeper rooting store or flows toward the discharge. Eventually, the water from the routing store will also feed the discharge. The ability of the production and routing stores to deliver water depends on their respective maximum capacity (in $\mathrm{mm}$ ), which are two parameters of the model. The other two parameters are the groundwater exchange coefficient (between the routing store and the ground) and the unit hydrograph (in days), controlling the time lag between the rainfall event and the resulting streamflow peak. Catchment water exchange (i.e. water coming from another recharge area) is also possible and controlled by the groundwater exchange coefficient. This model requires evapotranspiration and rainfall data (Figures $3 a$ and $3 b$ ) to set the input of water in the ground and then optimizes the four parameters to reconstruct the discharge of the catchment. This optimization is constrained by the actual discharge data of the catchment of Fontaine de Vaucluse (Figure 3c). The amount of water stored in the ground is eventually the sum of the two groundwater stores (Figure 3d). This hydrological model is built at the scale of the catchment $\left(1160 \mathrm{~km}^{2}\right)$, which is wider than the sensitivity zone of the SG $\left(30 \mathrm{~km}^{2}\right.$ at most according to Figure $\left.2 \mathrm{~b}\right)$. The computed time series of water storage thickness can now be converted into gravity by multiplying it with the asymptotic values 
obtained in Figure 2b, that is $\mathrm{g}_{\mathrm{h}}=-3.5 \mathrm{~nm} \cdot \mathrm{s}^{-2} \cdot \mathrm{cm}^{-1}$ or $\mathrm{g}_{\mathrm{h}}=4.1 \mathrm{~nm} \cdot \mathrm{s}^{-2} \cdot \mathrm{cm}^{-1}$ for a layer of water located above or below the iOSG-24, respectively.
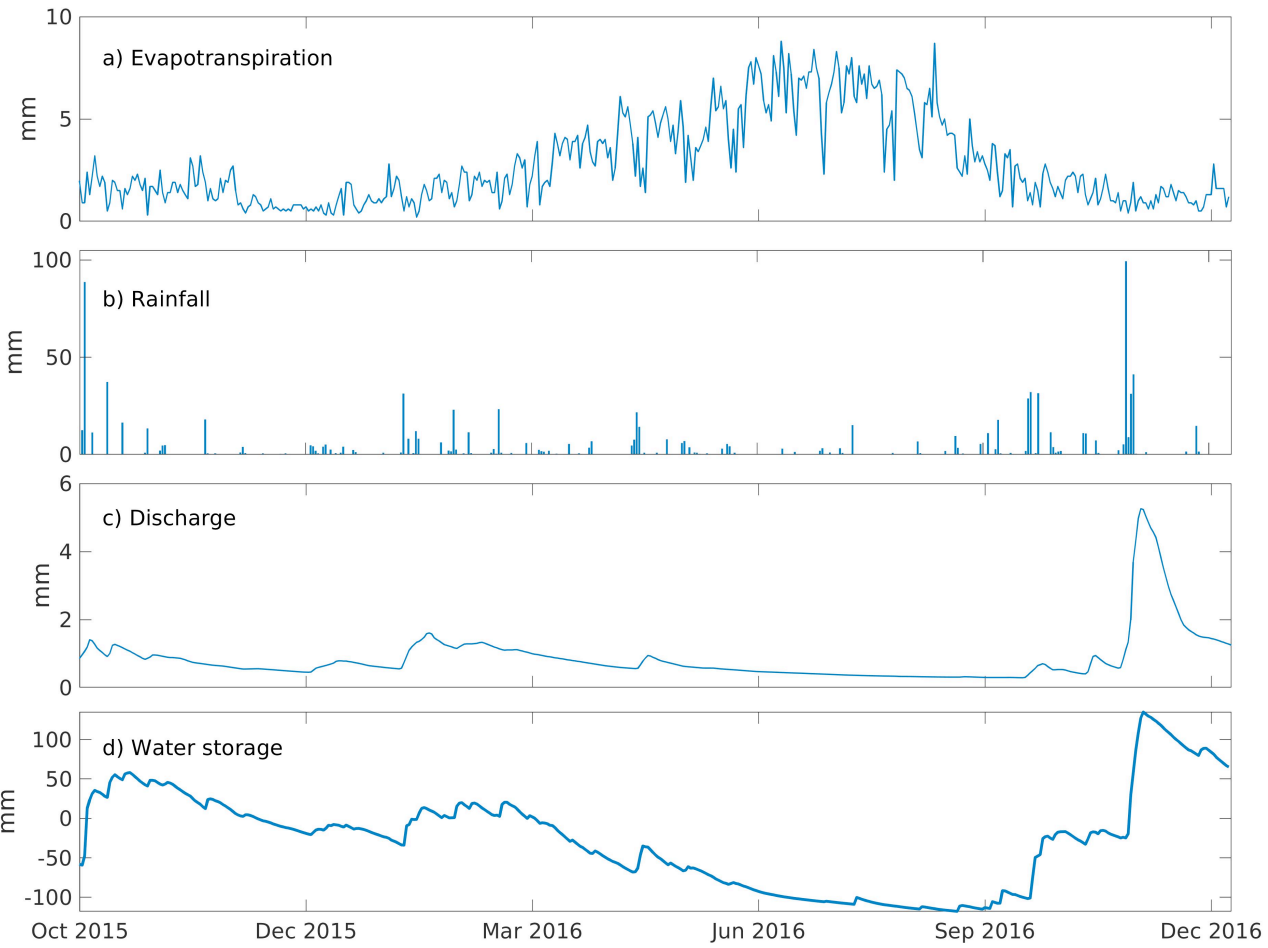

Fig. 3. Time series of a) the evapotranspiration, b) the rainfall and c) the discharge data d) the water storage thickness. This thickness can be multiplied by the area of the Fontaine de Vaucluse's catchment $\left(1160 \mathrm{~km}^{2}\right)$ to obtain the volume of water storage. The rainfall and evapotranspiration time-series that constrain this model are shorter than the iOSG-24 time series so that the computed water storage time series extent from October 2015 to December 2016.

\section{Results}

The best fit to the residual iOSG-24 time series is obtained when using $\mathrm{g}_{\mathrm{h}}=-3.5 \mathrm{~nm} . \mathrm{s}^{-2} . \mathrm{cm}^{-1}$ (Figure 4). This means that, at the time-scale of our measurements, the groundwater is mostly stored above the SG, in the karst unsaturated zone [7]. 


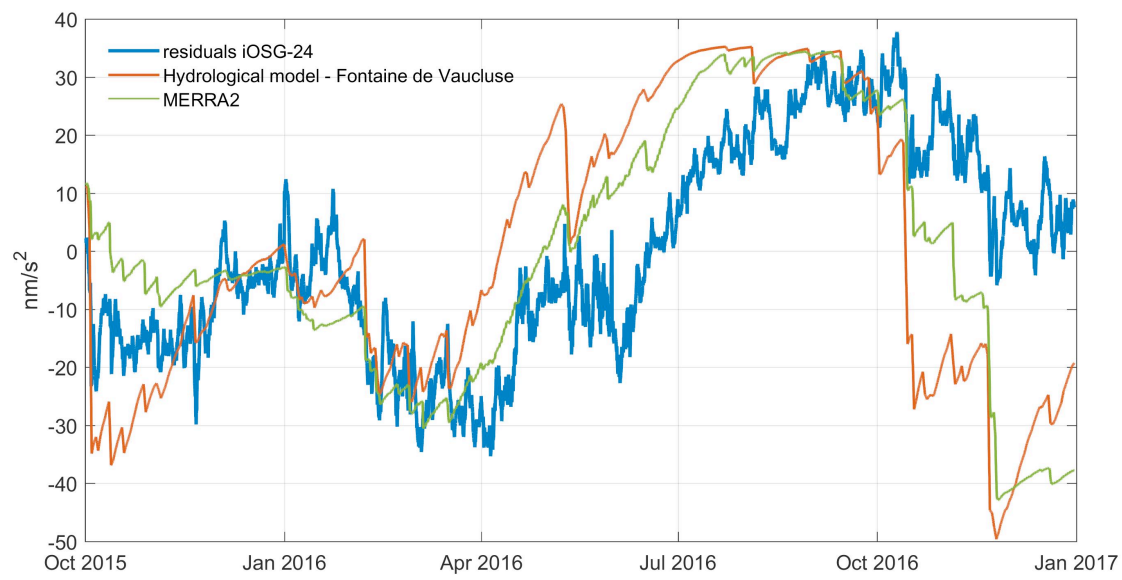

Fig. 4. Residual gravity time series recorded by iOSG-24 (blue line, same as Figure 1d) compared to the hydrological model computed for Fontaine de Vaucluse's catchment and converted into gravity for a layer of water located above the gravimeter (red line) and to the local hydro-gravity effect computed from MERRA2 hydrological model (green line).

This can be confirmed using the local hydro-gravity effect computed from MERRA2 hydrological model (Fig. 4 and [10]).

\section{Discussion}

We observe that both our hydrological model and MERRA2 show two similar biases:

1. An overestimation of the gravity effect (underestimation of the amount of water) from April to September 2016.

2. An underestimation of the gravity effect (overestimation of the amount of water) from October 2016 and January 2017.

Reasons for such misfits could be due to lateral fluxes of groundwater which bring more water above the SG than just rainfall and to rapid runoff at the surface hence letting only a fraction of the rainfall infiltrate the ground and alter the gravity. In addition, iOSG-24 is located on the border of the Fontaine de Vaucluse catchment. Therefore, water masses redistributed outside the catchment may also trigger gravity changes, yet they are not considered in our hydrological modelling. Differently, this misfit can also appear as a time lag between the hydrological model and the gravity residuals. Indeed, the latter are sensitive to the unsaturated zone only, while the hydrological model is constructed at the scale of the entire Fontaine de Vaucluse catchment, which aquifer includes both the unsaturated and saturated zone. Yet this catchment experiences variable time-lags between the filling of the unsaturated zone and that of the saturated zone [7]. Such hypotheses shall be further tested.

In this study, we have considered the average effect of water layer extending over rather large areas (circles with radii greater than $3 \mathrm{~km}$ ), maximizing the gravity effect on iOSG24. Consequently, the depth of this layer has a negligible effect on the resulting gravity effect. Thus, although we can conclude that water is stored mostly above the SG, we cannot assess the depth at which this storage occurs. This corresponds to an uncertainty of $\sim 500 \mathrm{~m}$ in vertical position. 
New perspectives are opened by the setting of a new SG (iGrav31) belonging to CRITEX (Innovative equipment for the critical zone) EquipEx at the surface of the LSBB, $~ 500 \mathrm{~m}$ above iOSG-24 and done in July 2018. Indeed, the layer of water stored will be observable by both instruments. After the rain, since water infiltrates the ground, we can expect to monitor a decrease of gravity in iGrav31 and simultaneously an increase of gravity in iOSG-24. Although this latter hypothesis needs to be numerically evaluated, it may provide new insights on the dynamics of groundwater infiltration.

\section{Conclusion}

The effect of seasonal groundwater redistribution is properly observed in the gravity timeseries recorded by the superconducting gravimeter iOSG-24. The gravity effect computed from global and local hydrological models suggest that groundwater is mostly stored above the gravimeter (i.e. within the karst hydrosystem unsaturated zone). Nevertheless, hydrogravity models and iOSG-24 residual gravity time series do not fit perfectly, suggesting that some of the groundwater masses and/or of the hydrological processes were not taken into account in our hypotheses. The setting of a second SG at the surface of the LSBB, $500 \mathrm{~m}$ above iOSG-24, scheduled for July 2018, will offer a unique SG configuration for better identifying hydrological processes occurring in this $500 \mathrm{~m}$-thick sample of karst system.

The iOSG-24 gravimeter of the LSBB was funded by the EQUIPEX MIGA (Matter wave-laser based Interferometer Gravitation Antenna) ANR-11-EQPX-0028 and by the European FEDER 2006-2013 "PFM LSBB - Développement des qualités environnementales du LSBB". We thank the reviewer for his constructive comments.

\section{References}

[1] J. M. Goodkind, “The superconducting gravimeter," Rev. Sci. Instrum., vol. 70, no. 11, pp. 4131-4152, 1999.

[2] B. Ducarme, A. P. Venedikov, J. Arnoso, X. D. Chen, H. P. Sun, and R. Vieira, "Global analysis of the GGP superconducting gravimeters network for the estimation of the pole tide gravimetric amplitude factor," J. Geodyn., vol. 41, no. 1, pp. 334-344, 2006.

[3] S. Rosat, J. Hinderer, and L. Rivera, "First observation of $2 \mathrm{~S} 1$ and study of the splitting of the football mode 0S2 after the June 2001 Peru earthquake of magnitude 8.4," Geophys. Res. Lett., vol. 30, no. 21, p. 2111, 2003.

[4] J.P. Montagner et al., "Prompt gravity signal induced by the 2011 Tohoku-Oki earthquake," Nat. Commun., vol. 7, pp. 1-7, 2016.

[5] B. Hector et al., "Hydro-gravimetry in West-Africa: First results from the Djougou (Benin) superconducting gravimeter," J. Geodyn., vol. 80, pp. 34-49, 2014.

[6] D. Crossley, J. Hinderer, and U. Riccardi, "The measurement of surface gravity.," Rep. Prog. Phys., vol. 76, no. 4, p. 046101, Apr. 2013.

[7] S. D. Carrière et al., "The role of porous matrix in water flow regulation within a karst unsaturated zone: an integrated hydrogeophysical approach," Hydrogeol. J., vol. 24, no. 7, pp. 1905-1918, 2016.

[8] L. Longuevergne et al., "Local and global hydrological contributions to gravity variations observed in Strasbourg," J. Geodyn., vol. 48, no. 3-5, pp. 189-194, Dec. 2009.

[9] T. Jacob et al., “Absolute gravity monitoring of water storage variation in a karst 
aquifer on the larzac plateau (Southern France)," J. Hydrol., vol. 359, no. 1-2, pp. 105-117, Sep. 2008.

[10] S. Rosat et al., "A two-year analysis of the iOSG-24 superconducting gravimeter at the low noise underground laboratory (LSBB URL) of Rustrel, France:

Environmental noise estimate," J. Geodyn., vol. 119, pp. 1-8, 2018.

[11] M. Van Camp and P. Vauterin, "Tsoft: graphical and interactive software for the analysis of time series and Earth tides," Comput. Geosci., vol. 31, no. 5, pp. 631640, Jun. 2005.

[12] J.-P. Boy and J. Hinderer, "Study of the seasonal gravity signal in superconducting gravimeter data," J. Geodyn., vol. 41, no. 1-3, pp. 227-233, Jan. 2006.

[13] R. H. Reichle et al., "Assessment of MERRA-2 Land Surface Hydrology Estimates," J. Clim., vol. 30, no. 8, pp. 2937-2960, Jan. 2017.

[14] N. Lesparre et al., "New insights on fractures deformation from tiltmeter data measured inside the Fontaine de Vaucluse karst system," Geophys. J. Int., vol. 208, no. 3, pp. 1389-1402, 2017.

[15] D. Nagy, "The prism method for terrain corrections using digital computers," Pure Appl. Geophys., vol. 63, no. 1, pp. 31-39, 1966.

[16] C. Perrin, C. Michel, and V. Andréassian, "Improvement of a parsimonious model for streamflow simulation,” J. Hydrol., vol. 279, no. 1, pp. 275-289, 2003. 